\title{
Sobrevida e características ecocardiográficas de gatos com e sem cardiomiopatias*
}

\section{Survival and echocardiographic characteristics of cats with and without cardiomyopathies}

\author{
Carolina Deuttner Neumann Barroso, ${ }^{* *}$ Eriane de Lima Caminotto, ${ }^{* * *}$ Alan Calahani, ${ }^{* * * *}$ Matheus Folgearini Silveira**
}

\begin{abstract}
Resumo
Cardiopatias felinas apresentam importante relevância na rotina veterinária, todavia os seus aspectos epidemiológicos em gatos não são conhecidos regionalmente. O objetivo do estudo foi descrever a frequência das cardiopatias na região Norte e Vale do Itajaí no estado de Santa Catarina e determinar a sobrevida de pacientes cardiopatas e não cardiopatas. O estudo contou com a análise retrospectiva de 74 laudos ecocardiográficos e prontuários médicos de gatos oriundos de estabelecimentos veterinários da região, de janeiro de 2017 a dezembro de 2019. Tutores ou veterinários responsáveis foram contatados para averiguar a sobrevida dos animais. Os animais do estudo em sua maioria eram machos $(n=40 / 74)$ e sem raça definida $(n=47 / 74)$. Cardiomiopatia foi o diagnóstico mais comum $(n=21 / 74)$, com destaque para o fenótipo hipertrófica $(n=13 / 21)$. As cardiomiopatias foram diagnosticadas mais comumente em gatos acima de oito anos de idade. Os principais sinais clínicos nos gatos sintomáticos $(n=41 / 74)$ foram sopro ( $n=15 / 41)$ e dispneia $(n=6 / 41)$. Os principais achados ecocardiográficos foram hipertrofia concêntrica da parede livre do ventrículo esquerdo $(n=18 / 41)$ e dilatação do átrio esquerdo $(n=12 / 41)$. A mediana de sobrevida dos 74 gatos foi de $303 \pm 209.8$ dias, estando altamente relacionado com a classe do estadiamento clínico $(P=0,006)$. Gatos com fenótipo dilatada tiveram menor média de sobrevida (180.5 dias). As doenças concomitantes mais observadas foram doença renal crônica $(n=7 / 15)$, hipertensão $(n=5 / 15)$ e/ou hipertireoidismo $(n=3 / 15)$. Gatos com cardiomiopatias, sintomáticos e com estágios mais avançados de remodelamento cardíaco, demostraram viver menos se comparados com aqueles em estágio inicial da cardiopatia. Bem como pacientes com doenças de base associada apresentaram menor expectativa de vida.
\end{abstract}

Palavras-chave: Cardiomiopatias, Estadiamento, Felinos, Mortalidade.

\begin{abstract}
Feline cardiopathies are relevant on veterinary practice although lack of regional epidemiogical description. The purpose of this study is to determine cardiopathy prevalence and survival of cardiac and non-cardiac patients on Santa Catarina's northern and Itajai valley regions. The retrospective study included 74 echocardiographic exams and medical records from January 2017 to December 2019. Veterinarians and owners were contacted to check survival on cardiac and non-cardiac patients. The animals were most male $(n=40 / 74)$ and mongrel $(n=47 / 74)$. Cardiomyopathies were the most common diagnosis $(n=21 / 74)$, specially the hypertrophic phenotype $(n=13 / 21)$. The cardiomyopathy diagnosis was evidenced in cats above eight years old. The most usual clinical findings on symptomatic patients $(n=41 / 74)$ were cardiac murmur $(n=15 / 41)$ and dyspnea $(n=6 / 41)$. Left ventricular free wall concentric hypertrophy $(n=18 / 41)$ and left atrium dilation $(n=12 / 41)$ were the main echocardiographic findings. Median survival from 74 cats was $303 \pm 209,8$ days and related to clinical staging $(p=0,006)$. Cats with dilated cardiomyopathy phenotype presented lower mean survival (180,5 days). Concomitant diseases included chronic renal disease $(n=7 / 15)$, systemic arterial hypertension $(n=5 / 15)$ and/or hyperthyroidism $(n=3 / 15)$. In conclusion, cats with cardiomyopathies, symptomatic and with more advanced stages of cardiac remodeling, have shown to live less than those in early stage of heart disease. As well as patients with associated concomitant diseases, they had a lower life expectancy.
\end{abstract}

Keywords: Cardiomyopathy, Feline, Mortality Staging.

\section{Introdução}

As cardiopatias felinas possuem grande prevalência na prática clínica veterinária, sendo as cardiomiopatias as afecções cardiovasculares mais frequentes (Payne et al., 2015), tanto quanto as valvulopatias são para os cães (Mattin et al., 2015; Keene et al., 2019). As cardiomiopatias primárias adquiridas em gatos incluem os fenótipos hipertrófica $(\mathrm{CMH})$, restritiva (CMR), dilatada (CMD) e arritmogênica do ventrículo direito (CAVD) (Ferasin et al., 2003; Fuentes et al., 2020). Ademais,

*Recebido em 3 de setembro de 2020 e aceito em 2 de novembro de 2020.

**Discente Instituto Federal Catarinense (IFC) -campus Araquari. Araquari, Santa Catarina, Brasil.

***Docente Instituto Federal Catarinense (IFC) -campus Araquari. Araquari, Santa Catarina, Brasil.

****Médico Veterinário Cardiologista autônomo. Joinville, Santa Catarina, Brasil.

Autora para correspondência: carolina.deuttner@gmail.com. 
gatos podem desenvolver afecções miocáridicas secundárias a quadros inflamatórios ou desordens sistêmicas, como: hipertireoidismo, hipertensão arterial sistêmica e doença renal crônica (Riesen et al., 2007; Nakamura et al., 2011; Spalla et al., 2015), podendo não se enquadrar em nenhum fenótipo, sendo classificada como não específica (CMNE) (Fuentes et al., 2020).

As afecções miocárdicas estão associadas à disfunção contrátil dos cardiomiócitos e apresentam uma heterogeneidade nas suas várias apresentações, podendo haver sobreposição entre os fenótipos e remodelamento conforme a progressão da doença com o tempo, tornando-se um desafio diferenciá-las entre si (Fuentes et al., 2020). Como exemplo, um gato com $\mathrm{CMH}$ em estágio avançado pode apresentar aspectos tanto de CMR como CMD (Ferasin et al., 2003; Spalla et al., 2015).

O exame ecocardiográfico é o método de escolha disponível para diagnóstico e classificação das cardiomiopatias por permitir melhor visualização das alterações miocárdicas. Todavia, ainda há uma inconstância em distingui-las morfologicamente, sendo necessário aliar o diagnóstico a um criterioso exame clínico (Fuentes, 2015; Fox, Schober, 2015; Spalla et al., 2015; Fox et al, 2018). As manifestações clínicas mais comuns de pacientes cardiopatas consistem em sopros, sons de galope e dispneia (Duler et al, 2019). Contudo, o sopro pode ser auscultado em gatos saudáveis, sendo considerados funcionais, e não relacionados a uma alteração cardíaca em si (Coté et al., 2015). Da mesma forma, gatos podem apresentar-se assintomáticos ao longo da vida e desenvolver o quadro de morte súbita (Payne et al, 2015; Fox et al, 2018).

Portanto, em virtude da necessidade de determinar as características clínicas e epidemiológicas das cardiomiopatias em diversas regiões, o objetivo deste estudo foi relatar a frequência das cardiomiopatias na região Norte e Vale do Itajaí no estado de Santa Catarina e determinar a sobrevida e risco de mortalidade cardíaca em gatos.

\section{Materiais e métodos}

Foram selecionados gatos de diversas faixas etárias, pesos, raças e sexo, submetidos à avaliação cardíaca prévia a partir de encaminhamento clínico de outros profissionais da região Norte e Vale do Itajaí de Santa Catarina, no período de janeiro de 2017 a dezembro de 2019. Para a inclusão no estudo os indivíduos deveriam ter os dados de resenha, histórico clínico, terapêutica empregada e exame ecocardiográfico completos (Camacho e Mucha, 2014).

O exame ecocardiográfico foi realizado por um único médico veterinário cardiologista, com o auxílio de um aparelho de ultrassom (GE Vivid-e ${ }^{\circledR}$ ) e transdutor setorial adequado ao porte do animal. Os gatos permaneceram conscientes ao longo da avaliação e contidos em decúbito lateral, tanto direito quanto esquerdo, conforme as janelas acústicas desejadas. Para a captura das imagens ecocardiográficas, o exame foi realizado com os modos bidimensional, modo movimento, Doppler pulsado, contínuo, de fluxo em cores e tecidual, e os resultados das mensurações foram considerados a partir da média de três ciclos cardíacos consecutivos, de acordo com Boon (2011). Todos os parâmetros ecocardiográficos obtidos após a avaliação foram analisados de acordo com valores de referência para a espécie (Häggström et al., 2016) aliados aos critérios de classificação das cardiomiopatias (Fuentes et al., 2020).
A partir dos dados oriundos dos prontuários médicos e dos laudos ecocardiográficos, os gatos foram estadiados clinicamente com base na data do primeiro exame ecocardiográfico, sendo estabelecido como dia zero. Caso tenha sido realizado mais de uma avaliação cardiológica do mesmo paciente, foi considerado o primeiro encontro para a estimar a sobrevida. Os exames cardiológicos subsequentes foram empregados apenas para acompanhamento e evolução clínica do paciente.

No intuito de estabelecer parâmetros de sobrevida foi determinada a data do óbito estabelecida no prontuário clínico. Nos demais casos foi realizado o contato telefônico com o tutor cadastrado na ficha clínica e/ou veterinário responsável pelo animal, a fim de determinar se o indivíduo permanecia vivo ou se ocorreu o óbito do mesmo. Da mesma forma, o tutor foi questionado sobre quando ocorreu o óbito e se foi de origem cardíaca ou não cardíaca. Caso o tutor não soubesse informar a causa do óbito ou os dados da avaliação cardiológica estivessem ausentes em algum critério, os indivíduos eram removidos do estudo. Foi estabelecido o mês de junho do ano de 2020 como data final para a definição do status de sobrevida.

Para fins de análise estatística, foi empregado o teste de Shapiro-Wilk para investigar a normalidade dos dados. Os dados paramétricos foram comparados por meio do ANOVA seguido do teste de Tukey, enquanto os não paramétricos foram comparados com o teste de Kruskal Wallis, seguido do pós-teste de Dunn. No intuito de determinar a relação entre as variáveis foi realizada a correlação de Pearson e Spearman de acordo com a natureza dos dados. A partir dos critérios clínicos e ecocardiográficos foram determinadas curvas de KaplanMeier para a análise da sobrevida validado pelo teste Log-rank. A significância estatística foi atribuída a partir dep<0,05. 0 software RStudio foi empregado para a análise estatística dos dados obtidos.

Esse estudo foi aprovado pelo Comitê de Ética de Pesquisa com Seres Humanos (CEPSH) do Instituto Federal Catarinense - Araquari, sob o parecer N No. 3.985.758.

\section{Resultados}

\section{Característica Populacional}

Durante o período do estudo, 140 prontuários foram avaliados, dos quais 74 pacientes preencheram integralmente todos os critérios de inclusão, sendo possível averiguar seu histórico clínico, exame ecocardiográfico e estimar sua sobrevida. Pacientes com doenças de base incluíram 15/74 gatos, previamente diagnosticados com doença renal crônica $(n=7 / 15)$, hipertensão $(n=5 / 15)$ e/ou hipertireoidismo $(n=3 / 15)$. A maioria dos pacientes analisados foram machos $(n=40 / 74)$ e sem raça definida $(n=47 / 74)$, seguidos pelas raças Persa $(n=17 / 74 ;)$, Siamês $(n=8 / 74)$ e Scottish Fold $(n=2 / 74)$. A faixa etária e 0 peso dos felinos, no momento do exame cardiológico, foi de $8,5 \pm 6$ anos e $3,9 \pm 1,2$ quilos, respectivamente.

\section{Sinais Clínicos Prévios}

A maioria dos pacientes felinos eram sintomáticos $(n=41 / 74$; $55,4 \%)$. Os principais sinais clínicos incluíram sopro $(n=15 / 41)$, dispneia $(n=6 / 41)$, efusão pleural $(n=5 / 41)$ e tosse $(n=2 / 41)$. Os graus de intensidade do sopro, em uma escala de 1 a 6 , foram distribuídos em grau $2(n=4$, sendo que três desses eram 
sopros fisiológicos), $3(n=6), 4(n=3)$ e $5(n=2)$, todos com maior prevalência na auscultação em bordo esternal esquerdo. Apenas um animal apresentou pulso jugular positivo. Três dos 41 gatos apresentaram ascite, sendo dois de origem cardiogênica.

\section{Alterações Ecocardiográficas}

Dentre as alterações morfológicas encontradas, no que tange a região ventricular de pacientes sintomáticos, constatou-se, mais comumente, hipertrofia da parede livre do ventrículo esquerdo $(n=18 / 41)$ e espessamento do septo interventricular de modo assimétrico $(n=10 / 41)$, bem como hipertrofia dos músculos papilares do ventrículo esquerdo $(n=5 / 41)$. Em relação aos átrios, visibilizou-se aumento moderado a grave da câmara atrial esquerda ( $n=12 / 41)$ e/ou aumento biatrial $(n=6 / 41)$. Não foram visibilizados trombos intracavitários nos pacientes estudados. Disfunção diastólica $(n=6 / 41)$ e sistólica $(n=3 / 41)$ também foram diagnosticados. Insuficiências valvares foram perceptíveis nas valvas mitral $(n=8 / 41)$, tricúspide $(n=5 / 41)$ e aórtica $(n=1 / 41)$, assim como o movimento sistólico anterior foi visível em três de 41 gatos. Nos gatos assintomáticos $(n=33 / 74)$ não foi observado remodelamento cardíaco significativo nas câmaras atriais e ventriculares.

\section{Incidência e risco para morbidade e mortalidade cardíaca}

Cardiomiopatias foi o diagnóstico mais comum encontrado na população de gatos cardiopata $(n=21 / 26)$, com destaque para a CMH e CMNE (Tabela 1). Cardiopatias congênitas também foram diagnosticadas, porém com menor prevalência $(n=5 / 26)$, sendo que dois animais apresentaram defeito do septo interventricular na porção perimembranosa $(n=2 / 5)$, seguido de displasia mitral $(n=1 / 5)$, displasia tricúspide $(n=1 / 5)$ e estenose aórtica $(n=1 / 5)$. As características populacionais das cardiopatias encontram-se na tabela 1.
Não houve diferença significativa entre a raça $(p=0,71)$ e o sexo $(p=0,22)$ dos animais com a presença de cardiomiopatia. Porém, cardiomiopatias foram diagnosticadas mais comumente em gatos de meia idade acima de oito anos $(P=0,04)$. Em relação a sobrevida desses gatos, a presença da cardiomiopatia pode ter forte influência no prognóstico do paciente, contudo, não se mostrou significativa (Figura 1).

Figura 1: Curvas de Kaplan-Meier (validado pelo teste Log-rank) com aspecto de sobrevida dos 74 felinos correlacionada com a presença de cardiomiopatia no paciente

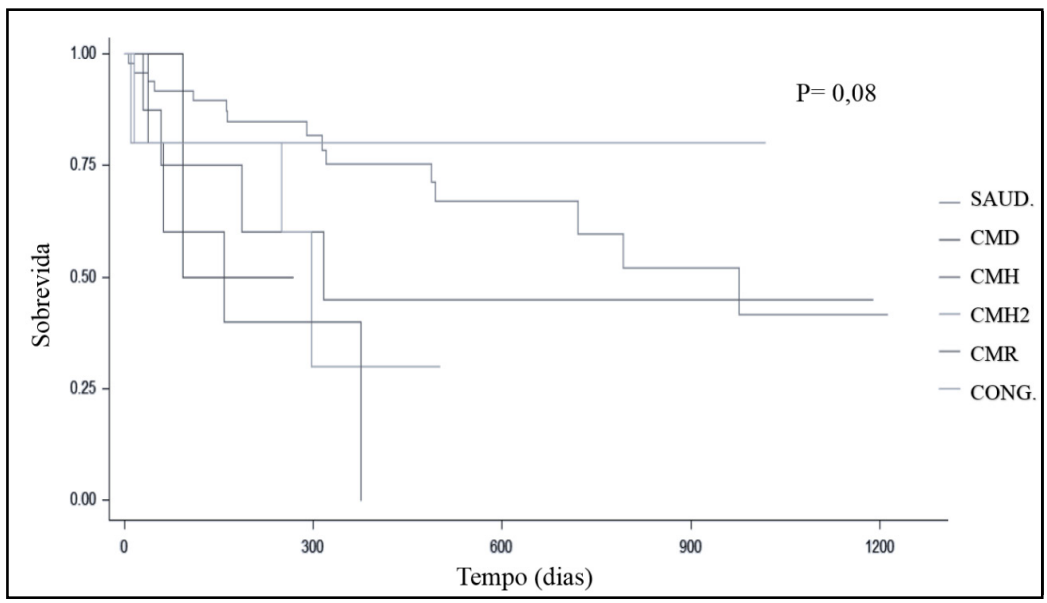

SAUD. - Saudáveis. CMD - Cardiomiopatia Dilatada. CMH - Cardiomiopatia hipertrófica. $\mathrm{CMH} 2$ - Cardiomiopatia Hipertrófica associada à doença de base. CMR - Cardiomiopatia Restritiva. CONG. - Congênitas.

Até o momento do encerramento do estudo, 29/74 (39.1\%) gatos vieram a óbito, sendo que 15/29 (51.7\%) morreram por complicações cardíacas $(\mathrm{P}<0,001)$. A mediana de sobrevida para todos os 74 gatos foi de $303 \pm 210$ dias, estando altamente relacionado com a classe do estadiamento clínico (Figura 2, Tabela 2). Em relação a prescrição medicamentosa, gatos cardiopatas e/ou com doença de base passaram a receber protocolos terapêuticos variados no momento do exame cardiológico, como consta na tabela 3.

Tabela 1: Características populacionais e de sobrevida das cardiopatias dos 74 gatos.

\begin{tabular}{|c|c|c|c|c|c|c|}
\hline Cardiopatia & $\mathrm{Ne} \%$ & Sexo & Raça & $\begin{array}{l}\text { Idade e Desvio } \\
\text { Padrão (meses) }\end{array}$ & $\begin{array}{l}\text { Sobrevida } \\
\text { média (dias) }\end{array}$ & Estadiamento \\
\hline $\mathrm{CMH}$ & $13 / 74(17.5 \%)$ & $\begin{array}{l}M(10 / 13) \\
F(3 / 13)\end{array}$ & $\begin{array}{l}\text { SRD (7/13), Persa }(5 / 13) \\
\text { Scottish Fold }(1 / 13)\end{array}$ & $114 \pm 59.3$ & $366,75^{\star}$ & B1 (8/13), B2 (4/13), C (1/13) \\
\hline CMNE & $5 / 74(6.75 \%)$ & $\begin{array}{l}M(1 / 5) \\
F(4 / 5)\end{array}$ & $\begin{array}{l}\text { SRD }(3 / 5), \text { Persa }(1 / 5) \\
\text { Siamês }(1 / 5)\end{array}$ & $180 \pm 46.8$ & 188.4 & B2 $(5 / 5)$ \\
\hline CMD & $2 / 74(2.70 \%)$ & $\begin{array}{l}M(1 / 2) \\
F(1 / 2)\end{array}$ & $\operatorname{SRD}(2 / 2)$ & $144 \pm 84.8$ & 180.5 & B2 (1/2), D (1/2) \\
\hline CONG. & $5 / 74(6.75 \%)$ & $\begin{array}{l}M(4 / 5) \\
F(1 / 5)\end{array}$ & SRD (4/5) Siamês (1/5) & $24 \pm 33.8$ & 526.8 & B1 (4/5), B2 (1/5) \\
\hline
\end{tabular}

CMH - Cardiomiopatia Hipertrófica, CMNE - Cardiomiopatia Não Específica, CMD - Cardiomiopatia Dilatada, CMR - Cardiomiopatia Restritiva, CONG. - Congênitas, SRD - Sem Raça Definida, M - Macho, F - Fêmea.

*264,2 dias se associado a doença de base. 
Figura 2: Curvas de Kaplan-Meier (validado pelo teste Log-rank) para a análise da sobrevida dos 74 felinos do estudo correlacionada com a classe de estadiamento do paciente (Fuentes et al., 2020)

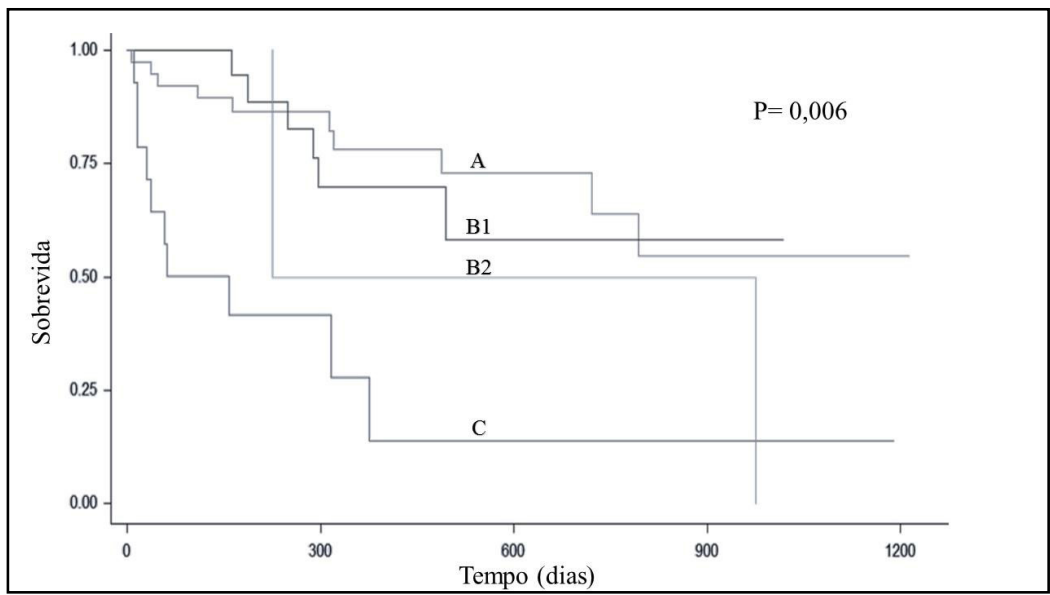

Tabela 2: Classificação do estadiamento dos pacientes felinos correlacionados com a mediana da sobrevida e dos parâmetros ecocardiográficos

\begin{tabular}{lccccc}
\hline Classificação & $\mathrm{Ne} \%$ & Sobrevida (dias) & $\mathrm{AE}(\mathrm{mm})$ & $\mathrm{AE}: \mathrm{Ao}(\mathrm{mm})$ & $\mathrm{SIVd}(\mathrm{mm})$ \\
\hline A & $38 / 74(51.4 \%)$ & $331,5 \pm 342$ & $11,95 \pm 1,72$ & $0,87 \pm 0,12$ & $3,8 \pm 0,66$ \\
B1 & $19 / 74(25.7 \%)$ & $357 \pm 235$ & $13,3 \pm 1,59$ & $1,08 \pm 0,16$ & $4,1 \pm 0,96$ \\
B2 & $14 / 74(18.9 \%)$ & $144 \pm 357$ & $16 \pm 3,36$ & $1,16 \pm 0,45$ & $4,5 \pm 1,4$ \\
C & $2 / 74(2.7 \%)$ & $225 \pm 152$ & $15,8 \pm 0,56$ & $1,49 \pm 0,27$ & $5,2 \pm 2,4$ \\
D & $1 / 74(1.4 \%)$ & 93 & 14,2 & 1,94 & 4,6 \\
Total geral & 74 & 303 & - & - & - \\
\hline
\end{tabular}

AE - Átrio Esquerdo, AE:Ao - Relação Átrio Esquerdo e Aorta, SIVd - Espessura do septo interventricular em diástole.

Tabela 3: Fármacos prescritos para pacientes cardiopatas e/ou com doenças de base associada após o exame ecocardiográfico

\begin{tabular}{lccc}
\hline \multicolumn{1}{c}{ Medicamento } & Cardiopatas & Não Cardiopatas & Total \\
\hline Pimobendan & 5 & 0 & $\mathrm{n}=5$ \\
Clopidogrel & 6 & 0 & $\mathrm{n}=6$ \\
Furosemida & 2 & 0 & $\mathrm{n}=2$ \\
Sildenafil & 1 & 0 & $\mathrm{n}=1$ \\
Beta bloqueador & 7 & 2 & $\mathrm{n}=9$ \\
Inibidor de ECA & 2 & 0 & $\mathrm{n}=2$ \\
Anlodipino & 0 & 2 & $\mathrm{n}=2$ \\
Metimazol & 3 & 0 & $\mathrm{n}=3$ \\
Total & $\mathrm{n}=26$ & $\mathrm{n}=4$ & $\mathrm{n}=30$ \\
\hline
\end{tabular}

ECA - Enzima Conversora de Angiotensina. 
Considerando a fisiopatogenia das cardiomiopatias, parâmetros diagnósticos ecocardiográficos como o diâmetro de câmara atrial esquerda $(>10,8 \mathrm{~mm})$, septo interventricular em diástole $(>3,9 \mathrm{~mm})$ e razão átrio esquerdo:aorta $(>1,14)$ foram definidos como critérios de evolução negativa. Todavia os parâmetros de diagnóstico não foram significativos para determinar a sobrevida (Figura 3).

Figura 3: Curvas de Kaplan-Meier (validado pelo teste de Long-rank) com os aspectos de sobrevida de 74 gatos do estudo correlacionados aos parâmetros ecocardiográficos (A) AE, (B) SIVd, (C) AE:Ao.
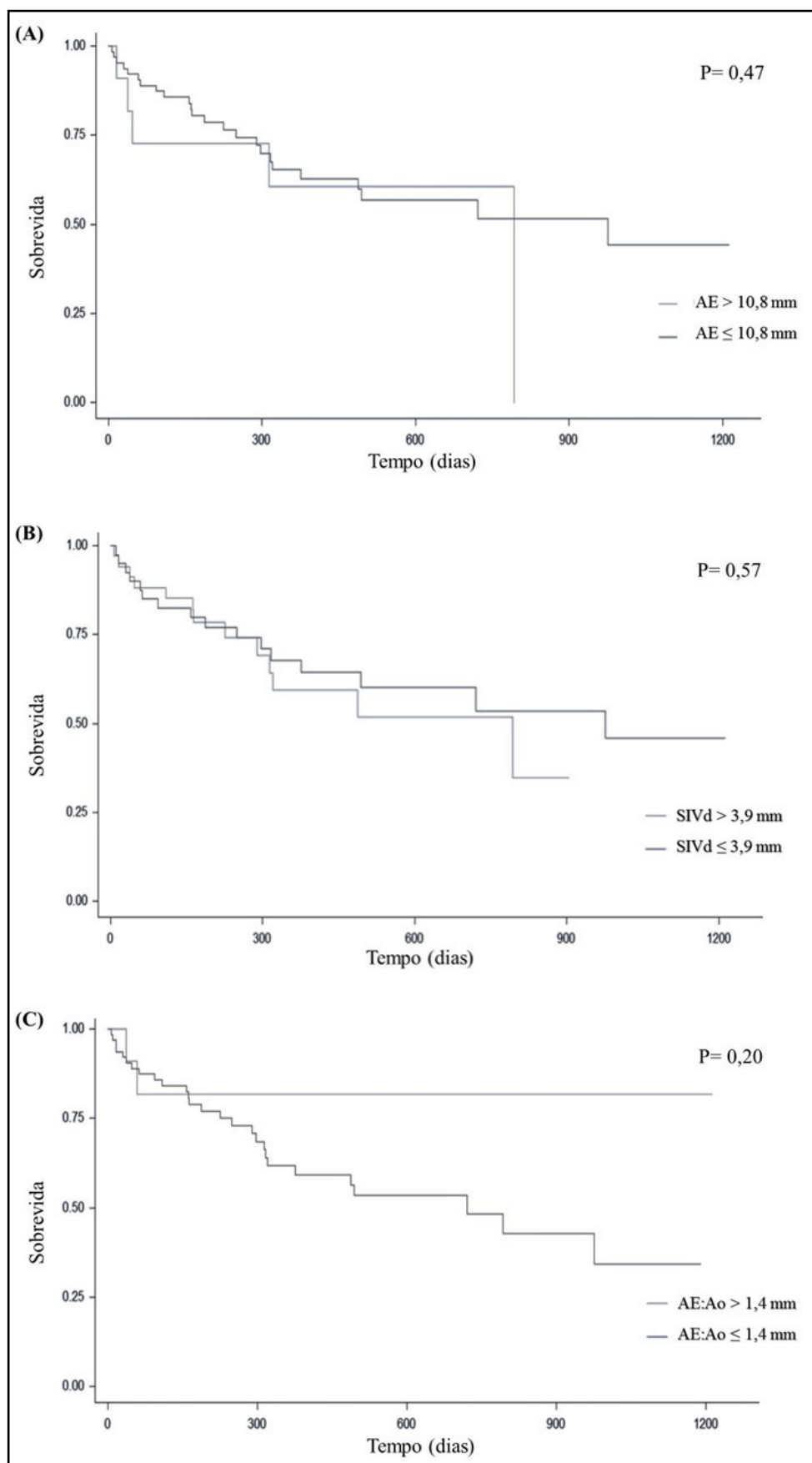

$\mathrm{AE}$ - Átrio Esquerdo, AE:Ao - Relação Átrio Esquerdo e Aorta, SIVd - Espessura do septo interventricular em diástole.

\section{Discussão}

Os resultados deste estudo demonstraram que a principal afecção cardíaca em gatos foram as cardiomiopatias, com maior relevância o fenótipo hipertrófica conforme observado em estudos anteriores (Ferasin et al., 2003; Spalla et al., 2015; Fox et al., 2018). Uma pesquisa na região sul brasileira relatou que doenças do sistema cardiovascular representaram a sexta maior causa de morte entre felinos (Rolim, 2017), todavia, este estudo foi o primeiro a estimar a sobrevida de pacientes com cardiomiopatias, assim como descrever seus aspectos ecocardiográficos. Durante o estudo foi possível averiguar que doenças cardíacas congênitas também se fizeram presentes na população felina local, porém trata-se de alterações raras (Tidholm et al., 2014; Schrope, 2015).

Os gatos diagnosticados com cardiomiopatias em sua maioria eram sem raça definida, inclusive os animais enquadrados nos fenótipos hipertrófico e dilatado, contrariando o observado na literatura (Ferasin et al, 2003; Trehiou-sechi et al., 2012). Algumas raças estão mais predispostas para desenvolverem cardiomiopatias, sendo a raça Persa com maior prevalência para CMH e CMD (Ferasin et al, 2003; Meurs et al., 2005; Meurs et al., 2007; Chetboul et al., 2012; Trehiousechi et al., 2012; März et al, 2015). Gatos machos mostram maior predominância para $\mathrm{CMH}$ (Spalla et al., 2015; Fox et al., 2018), mas neste estudo não relatou predisposição sexual para a presença das cardiomiopatias. A idade, contudo, em que comumente se diagnosticam as cardiomiopatias vai de encontro com dados já publicados (Coté, 2011; Trehiou-sechi et al., 2012; Spalla et al., 2015; Chetboul et al., 2019), cujos gatos acabam desenvolvendo a doença com seu envelhecimento, manifestando-a em torno dos oito anos de idade. Todavia, vale ressaltar que o fenótipo hipertrófica já foi diagnosticado em gatos jovens desde três meses de idade até dezessete anos, podendo ser considerada uma cardiomiopatia primária de origem genética (Rush et al., 2002; Ferasin et al., 2003; Mary et al., 2010; Silverman et al., 2012). Com base nessa amplitude etária, levantamentos epidemiológicos, como este estudo, demonstram um padrão populacional similar como visto em outros países ao relacionar o fenótipo hipertrófica e a idade dos gatos (Ironside et al., 2020).

Sabe-se que gatos podem desenvolver cardiomiopatias secundárias a desordens sistêmicas, especialmente no hipertireoidismo, hipertensão arterial sistêmica e doença renal crônica, que se não controladas com a terapia adequada podem progredir para uma insuficiência cardíaca congestiva (Riesen et al., 2007; Spalla et al., 2015; Duler et al., 2019). Essas comorbidades são associadas, mais comumente, ao fenótipo $\mathrm{CMH}$ conforme observado nesse estudo. Dentre os pacientes estudados, aqueles com doenças de base recebiam protocolos terapêuticos variados, porém não foi possível acessar integralmente todos os prontuários médicos para saber quais medicamentos prévios estavam sendo administrados para correlacionar se tais dados interferiram na sobrevida desses gatos. 
Em relação a sobrevida, gatos sintomáticos diagnosticados com $\mathrm{CMH}$, em média, vivem 700 dias, sendo que, se assintomáticos, a média estende-se até cinco anos, e aqueles com quadros de insuficiência cardíaca congestiva ou tromboembolismo atrial instalados, varia de dois a seis meses (Atkins et al., 1992; Rush et al., 2002; Trehiou-Sechi et al., 2012; Schober et al., 2013; Fox et al., 2018; Romito et al., 2018;). Neste estudo, todos os gatos classificados nesse fenótipo eram sintomáticos e apresentaram uma sobrevida mediana abaixo daquela previamente reportada (366,75 dias). Isso pode estar relacionado tanto ao tempo de avaliação do estudo como a baixa quantidade de casos diagnosticados ( $n=5 / 13$ ) (Rush et al., 2002; Payne et al., 2013; Fox et al., 2018). Ao analisarmos os gatos com CMNE, estes viveram menos do que esperado de acordo com literatura. Isso pode estar associado ao fato dessa cardiopatia representar os pacientes que não se enquadram como portadores de nenhum fenótipo clássico específico, sendo descritos de acordo com a alteração morfológica e funcional presentes no miocárdio (Ferasin et al., 2003; Ferasin et al. 2009). E por apresentar características heterogêneas ao diagnóstico, torna-se impreciso determinar com acurácia a sobrevida desses pacientes.

Por sua vez, os gatos com CMD apresentaram uma sobrevida mediana aproximada de 180 dias. Em relação a sobrevida dessa enfermidade, pouco tem-se relatado (Pion et al., 1992; Ferasin et al., 2003; Ferasin et al., 2009; Hambrook e Benett, 2012), porém é importante ressaltar que a CMD pode estar relacionada desde a deficiências de taurina (Pion et al., 1992), como ser consequência de uma $\mathrm{CMH}$ ou CMR em estágios finais da doença (Ferasin et al., 2003; Spalla et al., 2015). Por outro lado, a sobrevida média para gatos com CMR é em torno de sete meses a um ano após o diagnóstico (Spalla et al., 2015; Chetboul et al., 2019). Todavia, apenas um paciente apresentou esse fenótipo no presente estudo e não se pode extrapolar os resultados do indivíduo em comparação com uma população de gatos com CMR.

É importante ressaltar que as cardiomiopatias possuem uma grande variabilidade fenotípica, e cuja evolução do quadro clínico pode levar a sobreposição de fenótipos. Isso dificulta estabelecer o tempo de sobrevida para cada cardiomiopatia, uma vez que diferenciá-las entre si mostra-se um desafio clínico. Ademais, como a classificação das cardiomiopatias em gatos deriva da medicina humana, pautada em evidências científicas (Konta et al., 2015; Fuentes et al., 2020), ainda temos lacunas ao nortear a conduta clínica veterinária frente às afecções miocárdicas que o consenso sanou paliativamente. Sendo assim, ainda são necessários estudos prospectivos e de demais naturezas para estabelecer fidedignamente as cardiomiopatias em gatos.

Pacientes com doença de base associada às cardiomiopatias como hipertensão arterial sistêmica, hipertireoidismo ou doença renal crônica viveram menos. Esse fato pode estar associado ao manejo terapêutico inadequado e a evolução natural da doença de base. No que tange a hipertensão arterial sistêmica, esta eleva a resistência vascular periférica, gerando remodelamento cardíaco ventricular de forma crônica (Chetboul el al., 2008). Por sua vez, na doença renal crônica, a progressão da lesão renal bem como a ativação neuroendócrina concomitante de forma compensatória, pode induzir o desenvolvimento de hipertensão arterial sistêmica (Bijsmans et al., 2015; Sparkes et al., 2016). Neste estudo, sete gatos, dentre os 12 animais tanto com hipertensão e/ou doença renal, foram classificados como pacientes cardiorrenais, dos quais cinco (de sete) apresentaram remodelamento cardíaco decorrente das comorbidades primárias.

Da mesma forma, gatos com hipertireoidismo apresentam anormalidades cardíacas, pois o excesso do hormônio tireoidiano altera o cronotropismo, dromotropismo e gera remodelamento miocárdico (Klein e Ojamaa, 2001; Vaske et al., 2014). Os gatos hipertireoideos estudados tiveram em comum o aumento do átrio esquerdo e do septo interventricular em diástole, repercutindo no fenótipo $\mathrm{CMH}$.

Em relação a terapêutica das doenças de base, terapias antihipertensivas, quando já há lesão em órgãos alvos, como no coração, não tem demonstrado um benefício significativo na sobrevida desses gatos (Jepson et al., 2007; Taylor et al., 2017). Igualmente, com a evolução da endocrinopatia, diminui-se a resposta do animal ao manejo medicamentoso, consequentemente, impacta em menor sobrevida desses animais (Carney et al., 2016; Watson et al, 2018). O manejo terapêutico de gatos cardiopatas varia de acordo com as alterações cardíacas encontradas e podem interferir na expectativa de vida dos pacientes. Estudos comprovaram que o uso de pimobendan e clopidogrel, aumentaram a sobrevida de gatos com cardiomiopatias e com risco de insuficiência cardíaca (Gordon et al., 2012; Doreste et al., 2014; Hogan et al., 2015). Por outro lado, o uso de beta bloqueadores, como o atenolol, por exemplo, não mostram interferir no prognóstico do paciente cardiopata (King et al., 2019). No nosso levantamento, os protocolos adotados pelos veterinários foram tabulados, mas desconsiderados ao estimar a sobrevida dos gatos, pois não foi possível ter acesso acurado ao acompanhamento terapêutico.

Foram estabelecidos valores de corte para parâmetros ecocardiográficos de relevância clínica a fim de determinar a influência na sobrevida (Finn et al., 2010; Häggström et al., 2016). Todavia, tais parâmetros não foram relevantes para estimar a sobrevida dos animais, sugerindo que mais estudos sejam feitos para tentar correlacionar mensurações ecocardiográficas com a expectativa de vida de felinos e não somente ao diagnóstico de enfermidades cardiovasculares

Por outro lado, se relacionarmos o estadiamento proposto por Fuentes e colaboradores (2020) das cardiomiopatias com a sobrevida dos gatos no estudo, nota-se que conforme agravase o remodelamento cardíaco, diminui-se a expectativa de vida desses pacientes. Nesse estudo, contudo, somente dois pacientes foram estadiados como classe $C$, sendo que a variação da sobrevida dos indivíduos pode ter influenciado nos resultados. Já os pacientes B2 foram laudados tanto com alterações cardíacas congênitas, quanto com cardiomiopatias secundária, não seguindo um padrão específico de remodelamento cardíaco, tornando difícil estabelecer uma característica populacional para esse estadiamento. Por conseguinte, estudos com pacientes de estágios terminais de cardiomiopatias são escassos e inacurados (Payne et al., 2013; Häggström et al., 2015; Fox et al., 2019).

Em relação aos sinais clínicos, os gatos que participaram do estudo apresentaram, em sua maioria, manifestações prévias condizentes com o relatado para pacientes cardiopatas (Nakamura et al., 2011; Coté et al., 2015; Duler et al, 2019). Casos mais graves estadiados em C e D, apresentaram insuficiência cardíaca congestiva e, consequentemente, tiveram reflexos negativos na sobrevida. 
Por fim, apesar de ser um levantamento regional, com número amostral reduzido, o estudo cumpre com as necessidades de embasamento clínico local, para demonstrar a prevalência de cardiomiopatias nos gatos e exemplificar a sobrevida desses animais. Mesmo sendo um estudo retrospectivo, cujo ideal para acompanhar o prognóstico dos pacientes, determinar sua sobrevida e associar o estadiamento clínico com os achados ecocardiográficos, seria acompanhá-los até seu óbito.

Com isso, conclui-se que ao se tratar da sobrevida de gatos com cardiomiopatias, pacientes sintomáticos e com estágios

\section{Referências}

ATKINS, C.E. et al. Risk factors, clinical signs, and survival in cats with a clinical diagnosis of idiopathic hypertrophic cardiomyopathy: 74 cases (1985-1989). Journal of American Veterinary Medical Association, v. 201, n. 4, p. 608-613, 1992.

BIJSMANS, E.S. et al. Changes in Systolic Blood Pressure over Time in Healthy Cats and Cats with Chronic Kidney Disease. Journal of Internal Medicine, v. 29, n. 3, p. 855-861, 2015.

BOON, J.A. Veterinary Echocardiography. 2 n. lowa: WileyBlackwell, 2011.

CAMACHO, A.A.; MUCHA, C. J. Semiologia do Sistema Circulatório de Cães e Gatos. In: FEITOSA, Francisco Leydson F. (org.). Semiologia veterinária: a arte do diagnóstico. São Paulo: Roca, 2014. p. $544-585$.

CARNEY, C.H. et al. AAFP Guidelines for the Management of Feline Hyperthyroidism. Journal of Feline Medicine and Surgery, v. 18, n. 5, p. 400-416, 2016.

CHETBOUL, V. et al. Spontaneous feline hypertension: clinical and echocardiographic abnormalities, and survival rate. Journal of Internal Medicine, v. 17, n. 1, p. 89-95, 2003.

CHETBOUL, V. et al. Prospective echocardiographic and tissue Doppler screening of a large Sphynx cat population: Reference ranges, heart disease prevalence and genetic aspects. Journal of Veterinary Cardiology, v. 14, p. 497-509, 2012.

CHETBOUL, $V$. et al. Clinical, epidemiological and echocardiographic features and prognostic factors in cats with restrictive cardiomyopathy: A retrospective study of 92 cases (2001-2015). Journal of Internal Medicine, v. 33, n. 3, p. 12221231, 2019.

COTÉ, E. et al. Feline Cardiology. Philadelphia: Wiley-Blackwell, 2011.

COTÉ, E. et al. Management of incidentally detected heart murmurs in dogs and cats. Journal of Veterinary Cardiology, v. 17, n. 4, p. 245-261, 2015.

DORESTE, R.Y. et al. Case-control study of the effects of pimobendan on survival time in cats with hypertrophic cardiomyopathy and congestive heart failure. Journal of the American Veterinary Medical Association, v.245, n. 5, p. 534539, 2014.

DULER, L.; SCOLLAN, K. F.; LEBLANC, N. L. Left atrial size and volume in cats with primary cardiomyopathy with and without congestive heart failure. Journal of Veterinary Cardiology, v. 24, p. 36-47, 2019.

FERASIN, L. Feline idiopathic cardiomyopathy: a retrospective study of 106 cats (1994-2001). Journal of Feline Medicine and Surgery, v. 5, n. 3, p. 151-159, 2003. mais avançados de remodelamento cardíaco, demonstram viver menos se comparados com aqueles em estágio inicial da cardiopatia. Além disso, pacientes com doenças de base associada possuem menor expectativa de vida. Para se estabelecer maiores relações entre a sobrevida dos gatos com cardiomiopatias e os parâmetros ecocardiográficos, são necessários outros estudos que associam o dimensionamento cardíaco com o prognóstico do paciente, não apenas correlacionando a classificação da cardiomiopatia com o risco de mortalidade nos gatos.

FERASIN, L. Feline myocardial disease: Classification, pathophysiology and clinical presentation. Journal of Feline Medicine and Surgery, v. 11, n. 3, p. 183-194, 2009.

FERASIN, L. Feline myocardial disease 2: Diagnosis, prognosis and clinical management. Journal of Feline Medicine and Surgery, v.11, n.3, p. 183-194, 2009

FOX, P. R.; SCHOBER, K.A. Management of asymptomatic (occult) feline cardiomyopathy: Challenges and realities. Journal of Veterinary Cardiology, v. 17, n. 1, p. 150-158, 2015.

FOX, P. R. et al. International collaborative study to assess cardiovascular risk and evaluate long-term health in cats with preclinical hypertrophic cardiomyopathy and apparently healthy cats: The REVEAL Study. Journal of Veterinary Internal Medicine, v. 32, n. 3, p. 930-943, 2018.

Fox, R. P. et al. Long-term incidence and risk of noncardiovascular and all-cause mortality in apparently healthy cats and cats with preclinical hypertrophic cardiomyopathy. Journal of Veterinary Internal Medicine, v. 33, n. 6, p. 2572-2586, 2019.

FUENTES, V. L. Heart disease: time to take cats seriously. Journal of Veterinary Cardiology, United Kingdom, v. 17, p. 2-5, August 2015. Editorial.

FUENTES, L. V. ACVIM consensus statement guidelines for the classification, diagnosis, and management of cardiomyopathies in cats. Journal of Veterinary Internal Medicine, v.34, n. 3, p. 10621077, 2020.

GORDON, G. S. et al. Effect of oral administration of pimobendan in cats with heart failure. Journal of the American Veterinary Medical Association, v. 241, n. 1, p. 89-94, 2012.

HAGGSTROM, J.; FUENTES, L. V.; WESS, G. Screening for hypertrophic cardiomyopathy in cats. Journal of Veterinary Cardiology, v. 17, n. 1, p. 134-149, 2015.

HAGGSTROM et al. Effect of Body Weight on Echocardiographic Measurements in 19.866 Pure Bred Cats with or without Heart Disease. Journal of Veterinary Internal Medicine, v.30, n.5, p. 1601-1611, 2016.

HAMBROOK, E. L., BENNETT, F. P. Effect of pimobendane on the clinical outcome and survival of cats with non-taurine responsive dilated cardiomyopathy. Journal of Feline Medicine and Surgery, v. 14, n. 4, p. 233-239, 2012.

HOGAN, D. F. et al. Secondary prevention of cardiogenic arterial thromboembolism in the cat: The double-blind, randomized, positive-controlled feline arterial thromboembolism; clopidogrel vs. aspirin trial (FAT CAT). Journal of Veterinary Cardiology, v. 17, p. 306-317, 2015.

IRONSIDE, V. A.; TRICKLEBANK, P. R.; BOSWOOD, A. Risk indictors in cats with preclinical hypertrophic cardiomyopathy: a prospective cohort study. Journal of Feline Medicine and Surgery, p. 1-11, 2020. 
JEPSON, R. E, ELIIOTT, J., BRODBELT, D. Effect of control of systolic blood pressure on survival in cats with systemic hypertension. Journal of Internal Medicine, v. 21, p. 402-409, 2007.

KEENE, B. W. et al. ACVIM consensus guidelines for the diagnosis and treatment of myxomatous mitral valve disease in dogs. Journal of Veterinary Internal Medicine, v. 33, n. 3, p.11271140, 2019.

KLEIN, I., OJAMAA, K. Thyroid Hormone and the Cardiovascular System. New England Journal of Medicine, v. 344, n. 7, p. 501-509, 2001.

KING, N. J. et al. Evaluation of benazepril in cats with heart disease in a prospective, randomized, blinded, placebo-controlled clinical trial. Journal of Veterinary Internal Medicine, v. 33, n. 6, p. 2559-2571, 2019.

KONTA, L., FRANKLIN, R. C., KASKI, J. P. Nomenclature and systems of classification for cardiomyopathy in children. Cardiol Young, v. 25, n. 2, p. 31-25, 2015.

Mary, J.; Chetboul, V.; Sampedrano, CC. Prevalence of the MYBPC3-A31P mutation in a large European feline population and association with hypertrophic cardiomyopathy in the Maine Coon breed. Journal of Veterinary Cardiology, v. 12, p. 155-161, 2010

MÄRZ, I. et al. Familial cardiomyopathy in Norwegian Forest cats Journal of Feline Medicine and Surgery, v. 17, n. 8, p. 681-691, 2015.

MATTIN, M.J. et al. Prevalence of and Risk Factors for Degenerative Mitral Valve Disease in Dogs Attending Primarycare Veterinary Practices in England. Journal of Veterinary Internal Medicine, v. 29, n. 3, p. 847-854, 2015

MEURS, K. M. et al. A cardiac myosin binding protein $\mathrm{C}$ mutation in the Maine Coon cat with familial hypertrophic cardiomyopathy. Human Molecular Genetics, v. 14, n. 23, p. 3587-3593, 2005.

MEURS, K. M. et al. A substitution mutation in the myosin binding protein $\mathrm{C}$ gene in ragdoll hypertrophic cardiomyopathy. Genomics, v. 90, n. 2, p. 261-264, 2007.

NAKAMURA, R. K. et al. Prevalence of echocardiographic evidence of cardiac disease in apparently healthy cats with murmurs. Journal of Feline Medicine and Surgery, v. 13, n. 4, p.266-271, 2011.

PAYNE, J. R. et al. Prognostic indicators in cats with hypertrophic cardiomyopathy. Journal of Internal Medicine, v. 27, n. 6, p. 14271436, 2013.

PAYNE, J. R.; BRODBELT, D. C.; FUENTES, V. L. Cardiomyopathy prevalence in 780 apparently healthy cats in rehoming centres (the CatScan study). Journal of Veterinary Cardiology, v. 17, p. 244-257, 2015.

PAYNE, J. R. et al. Risk factors associated with sudden death vs. congestive heart failure or arterial thromboembolism in cats with hypertrophic cardiomyopathy. Journal of Veterinary Cardiology, v. 17, p. 318-328, 2015.

PION, P. D. et al. Clinical findings in cats with dilated cardiomyopathy and relationship of findings to taurine deficiency. Journal of American Veterinary Medical Association, v. 15, p. $267-$ 285, 1992.

RIESEN, S.C. et al. Prevalence of heart disease in symptomatic cats: an overview from 1998 to 2005. Schweiz Arch Tierheilkd, v. 149, n. 2, p. 65-71, 2007
ROLIM, M. V. Causas de mortes em gatos na região sul do Brasil. Tese (Doutorado) - Universidade Federal do Rio Grande do Sul, Faculdade de Veterinária, Programa de Pós-Graduação em Ciências Veterinária, Porto Alegre, 2017.

ROMITO, G. et al. Diagnostic and prognostic utility of surface electrocardiography in cats with left ventricular hypertrophy. Journal of Veterinary Cardiology, v. 20, n. 5, p. 364-375, 2018.

RUSH, J. E. et al. Population and survival characteristics of cats with hypertrophic cardiomyopathy: 260 cases (1990-1999). Journal of the American Veterinary Medical Association, v. 220, n. 2, p. 202-207, 2002.

SPALLA, I. Survival in cats with primary and secondary cardiomyopathies. Journal of Feline Medicine and Surgery, v. 18, n. 6, p. 501-509, 2015.

SPARKES, H. A. et al. ISFM Consensus Guidelines on the Diagnosis and Management of Feline Chronic Kidney Disease. Journal of Feline Medicine and Surgery, v. 18, n. 3, p. 219-239, 2016.

SCHOBER, K. E. et al. Effect of treatment with atenolol on 5-year survival in cats with preclinical (asymptomatic) hypertrophic cardiomyopathy. Journal of Veterinary Cardiology, v. 15, n. 2, p. 93-104, 2013.

SCHROPE, D. P. Prevalence of congenital heart disease in 76,301 mixed-breed dogs and 57,025 mixed-breed cats. Journal of Veterinary Cardiology, v. 17, n. 3, p. 192-202, 2015.

TAYLOR, S. S. ISFM Consensus Guidelines on the Diagnosis and Management of Hypertension in Cats. Journal of Feline Medicine and Surgery, v. 19, n. 3, p. 288-303, 2017.

TIDHOLM, A. Congenital heart defects in cats: A retrospective study of 162 cats (1996-2013). Journal of Veterinary Cardiology, v. 17, p. 215-219, 2015.

TOGNI, M. et al. Causas de morte e razões para eutanásia em gatos na Região Central do Rio Grande do Sul (1964-2013). Pesquisa Veterinária Brasileira, v. 38, n. 4, p. 741-750, 2017.

TREHIOU-SECHHI, E. et al. Comparative Echocardiographic and Clinical Features of Hypertrophic Cardiomyopathy in 5 Breeds of Cats: A Retrospective Analysis of 344 Cases (2001-2011). Journal of Veterinary Internal Medicine, v. 26, p. 532-541, 2012.

VASKE, H. H. et al. Diagnosis and management of feline hyperthyroidism: current perspectives. Vet Med (Auckl), v. 20, n. 5, p. 85-96, 2014.

WATSON, N. et al. Clinicopathological features and comorbidities of cats with mild, moderate or severe hyperthyroidism: a radioiodine referral population. Journal of Feline Medicine and Surgery, v. 20, n. 12, p. 1130-1137, 2018. 